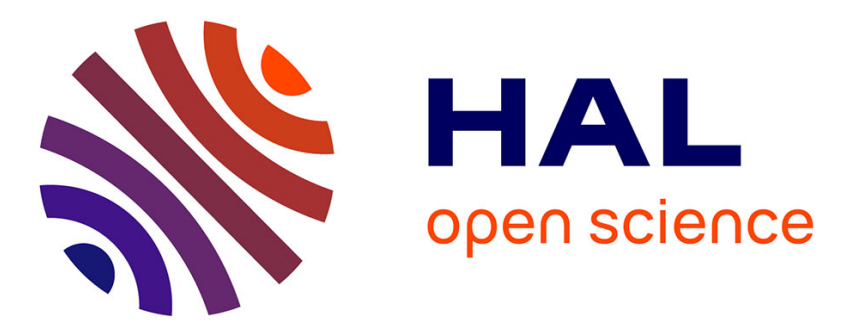

\title{
Etude par pompage de, charge des défauts induits à l'interface $\mathrm{Si}-\mathrm{SiO} 2$ par rayonnements ionisants
} Jean-Luc Autran, Bernard Balland, Jean-Pierre Vallard, Daniel Babot

\section{To cite this version:}

Jean-Luc Autran, Bernard Balland, Jean-Pierre Vallard, Daniel Babot. Etude par pompage de, charge des défauts induits à l'interface $\mathrm{Si}-\mathrm{SiO} 2$ par rayonnements ionisants. Journal de Physique III, 1994, 4 (9), pp.1707-1721. 10.1051/jp3:1994235 . jpa-00249219

\section{HAL Id: jpa-00249219 https://hal.science/jpa-00249219}

Submitted on 1 Jan 1994

HAL is a multi-disciplinary open access archive for the deposit and dissemination of scientific research documents, whether they are published or not. The documents may come from teaching and research institutions in France or abroad, or from public or private research centers.
L'archive ouverte pluridisciplinaire HAL, est destinée au dépôt et à la diffusion de documents scientifiques de niveau recherche, publiés ou non, émanant des établissements d'enseignement et de recherche français ou étrangers, des laboratoires publics ou privés. 
Classification

Physics Abstracts

$71.20-73.40 \mathrm{Q}-73.90$

\title{
Etude par pompage de charge des défauts induits à l'interface $\mathrm{Si}_{-} \mathrm{SiO}_{2}$ par rayonnements ionisants
}

\author{
Jean-Luc Autran ( $\left.{ }^{1}\right)$, Bernard Balland ( $\left.{ }^{1}\right)$, Jean-Pierre Vallard ( ${ }^{1}$ ) et Daniel Babot $\left({ }^{2}\right)$ \\ (1) Laboratoire de Physique de la Matière, Associé au Centre National de la Recherche \\ Scientifique, URA n 358, Institut National des Sciences Appliquées de Lyon, Bâtiment 502, \\ 20 avenue Albert Einstein, 69621 Villeurbanne Cedex, France \\ (2) Equipe Contrôle Non Destructif par Rayonnements Ionisants, Institut National des Sciences \\ Applıquées de Lyon, Bâtiment 303, 20 avenue Albert Einstein, 69621 Villeurbanne Cedex, \\ France
}

(Reçu le 28 mars 1994, accepté le 26 maı 1994)

\begin{abstract}
Résumé. - Nous avons étudié la réponse électrique des défauts de l'interface $\mathrm{Si}-\mathrm{SiO}_{2}$ $\langle 100\rangle$ présents dans des transistors MOS submicroniques après exposition à une source de rayonnement gamma $\left(\mathrm{Co}^{60}\right)$. La technique de pompage de charge à trois niveaux a permis de déterminer la distribution énergétique des paramètres des états d'interface (temps d'émission, sections efficaces de capture et densité d'états d'interface) dans la quasi-totalité de la bande interdite du silicium après différentes doses d'irradiation. Nous avons mis en évidence la possıbilité de caractériser, par pompage de charge classique et à trois nıveaux, les pièges d'oxyde proches de l'interface (états lents) induits par des rayonnements ionisants. Leur comportement en régime d'émıssion est ici examiné dans le cadre d'un mécanisme tunnel. Nous proposons une méthode originale, dérivée du pompage de charge à trois niveaux, pour déterminer la répartition spectrale de ces états lents.

Abstract. - We have studied electrical defects of $\langle 100\rangle \mathrm{Si}_{-} \mathrm{SiO}_{2}$ interface created by gamma rays $\left(\mathrm{Co}^{60}\right)$ in submicrometer MOS transistors. By means of three-level charge pumping, the energy distribution of interface-trap parameters (emission times, cross sections, state density) has been determined in most of the silicon bandgap after different irradiations. We have shown the possibility of characterizing, using standard and three-level charge pumping, the oxide traps near the interface (border traps) induced by ionizing radiations. Their behaviour in emission regime is here investigated in terms of tunneling mechanism. We propose a new method, based on the threelevel charge pumping technique, to determine the energy distribution of border traps.
\end{abstract}

\section{Introduction.}

L'exposition de structures métal-oxyde-semiconducteur (MOS) à des rayonnements ionisants conduit généralement à la création simultanée d'une charge électrique positive (fixe et permanente) piégée dans la couche d'oxyde et de défauts électriquement actifs à l'interface 
oxyde-semiconducteur [1-2]. Ces différents défauts jouent un rôle particulièrement important dans les composants électroniques car ils sont directement responsables des phénomènes d'instabilités électriques et de la dérive des paramètres clefs de fonctionnement des dispositifs, comme par exemple la tension de seuil des transistors. Les travaux de recherche actuels ont pour principal objet l'étude des propriétés structurales et électriques de ces défauts ainsi que de leurs mécanismes de création. Leur but est d'établir un modèle fiable de dégradation du système $\mathrm{Si}-\mathrm{SiO}_{2}$ dans des environnements radiatifs sévères, rencontrés dans les domaines des applications spatiales ou nucléaires. Dans ce travail, nous avons analysé la réponse électrique des défauts de l'interface $\mathrm{Si}-\mathrm{SiO}_{2}$ présents dans des transistors MOS submicroniques après exposition à une source de rayonnement gamma $\left(\mathrm{Co}^{60}\right)$. Pour ce faire, nous avons utilisé la technique de pompage de charge à trois niveaux dont nous avons récemment proposé une nouvelle instrumentation, bien adaptée aux composants de petites dimensions [3]. En utilisant différentes approches de pompage de charge, à deux niveaux $(2 \mathrm{CP})$ et à trois niveaux $(3 \mathrm{CP})$, nous avons déterminé les distributions énergétiques des temps d'émission, des sections efficaces de capture et de la densité d'états d'interface des pièges à électrons et à trous dans la bande interdite du silicium pour différentes doses d'irradiation des structures de test. Il nous a été possible de séparer les contributions des pièges d'interface ou «états rapides », à faible temps de réponse, et des pièges proches de l'interface ou «états lents », à grandes constantes de temps. Ces états sont associés à des défauts électriquement actifs, créés à l'interface et dans le volume de l'oxyde par les rayonnements ionisants. A l'aide d'un modèle très simple de mécanisme tunnel, le comportement des états lents en régime d'émission a été précisé de manière qualitative. Nous avons ensuite confronté l'ensemble de nos résultats aux travaux antérieurs de la littérature.

\section{Procédure expérimentale.}

2.1 Structures DE TEST. - Les structures utilisées sont des transistors MOS à canal N de longueur de canal submicronique (longueur effective $0,5 \mu \mathrm{m}$ ) issus d'un procédé de fabrication IBM CMOS. Ces transistors présentent une structure interdigitée : la source et le drain constituent une jonction unique en forme de peigne avec le substrat qui interpénètre le peigne de grille (surface de grille d"environ $12000 \mathrm{\mu m}^{2}$ ). Le substrat de silicium est une couche $\mathrm{P}$ épitaxiée, dopée au bore par implantation ionique, et déposée sur une plaquette de silicium $\mathrm{P}^{+}$obtenu par tirage Czochralski (orientation $\langle 100\rangle$ ). Les zones de type $\mathrm{N}$ sont obtenues par implantation et diffusion de phosphore. Une oxydation locale sur silicium permet l'isolation interdispositif. La résistivité du substrat ainsi que les tensions de seuil des dispositifs sont ajustées par un dopage additionnel de bore. Le dopage final moyen du substrat est donc, à la surface du canal, d'environ $10^{17} \mathrm{at} / \mathrm{cm}^{3}$. Les zones de source et de drain de type $\mathrm{N}^{+}$sont obtenues par implantation ionique et diffusion de phosphore et d'arsenic. Un procédé d'oxydation à $800^{\circ} \mathrm{C}$ conduit à un oxyde de grille de $14 \mathrm{~nm}$ d'épaisseur, de bonne qualité électrique. Le matériau de grille est du polysilicium, dopé au phosphore par implantation ionique, et d'épaisseur $460 \mathrm{~nm}$. Ce même matériau assure les contacts de source et de drain ; sa gravure est réalisée par plasma. Pour réduire sa résistivité, une couche de titane est déposée sur le polysilicium. Après un traitement thermique à $700{ }^{\circ} \mathrm{C}$, ces deux matériaux forment un composé $\mathrm{TiSi}_{2}$ qui possède une résistivité électrique 10 fois plus faible que le polysılicium. Enfin, une couche de $\mathrm{Si}_{3} \mathrm{~N}_{4}$ de $50 \mathrm{~nm}$ est déposée par dépôt chimique en phase vapeur à basse pression afin d'isoler les dispositifs de la couche finale de protection constituée d'un verre de silicate au bore et au phosphore d'épaisseur $650 \mathrm{~nm}$. Les contacts électriques sont ouverts à travers cette double couche isolante par gravure chimique et par dépôt de plots en aluminium. Différents traitements thermiques de passivation sous hydrogène au cours du procédé 
permettent d'abaisser la densité d'états d'interface à quelques $10^{10} \mathrm{eV}^{-1} \mathrm{~cm}^{-2}$ en fin de procédé.

2.2 Techniques utilisées. - Notre étude est basée sur l'utilisation de la technique du pompage de charge classique et d'une nouvelle méthode de pompage de charge à trois niveaux proposée récemment. L'instrumentation et la procédure d'acquisition de cette nouvelle approche ont été décrites en détail dans la référence [3]. Précisons que les performances (sensibilité et résolution en temps et en énergie) de cette méthode 3CP reposent sur l'emploi d'un générateur de signaux arbitraires (Lecroy 9100) extrêmement performant, ce qui permet de générer n'importe quelle forme d'onde avec une résolution temporelle de $5 \mathrm{~ns}$. Le remarquable profil des signaux ainsi obtenus [4], garantit un signal de mesure d'une grande stabilité. Comme nous l’avons proposé par ailleurs [5], la méthode de construction du signal à 3 niveaux de tension consiste à ne faire varier uniquement que les paramètres du troisième niveau (tension $V_{3}$ et durée $t_{3}$ ), les autres paramètres du signal appliqué sur la grille du transistor étant maintenus rigoureusement constants durant l'acquisition (durée et amplitude du signal en inversion et en accumulation, durée des différents temps de transition). Ainsi, à chaque valeur du temps intermédiaire $t_{3}$, la fréquence du signal est la plus élevée possible. Cette méthode, dite de la «fréquence ajustée», garantit une sensibilité maximale dans la mesure où la réponse en pompage de charge est proportionnelle à la fréquence $f$ du signal de mesure. La grandeur à considérer est alors la charge recombinée par période du signal de grille $Q_{\mathrm{t}}=I_{\mathrm{cp}} / f$, où $I_{\mathrm{cp}}$ est l'intensité du courant pompé (mesuré au niveau du substrat à l'aide d'un électromètre Keitley 617). La même instrumentation est utilisée pour les mesures en pompage de charge classique.

2.3 DétaILS EXPÉRIMENTAuX. - La dégradation des structures ainsi que l'ensemble des mesures ont été réalisés à température ambiante. Les irradiations par rayonnement gamma (énergies 1,17 et $1,32 \mathrm{Mev}$ ) ont été effectuées à l'aide d'une source au cobalt $\left(\mathrm{Co}^{60}\right)$ ) d'activité $20 \mathrm{Ci}$ sur des échantillons montés en boîtiers céramiques sans capot, à un débit de dose de $59 \mathrm{krad}$ par heure; les contacts électriques (source, drain, grille et substrat) étant maintenus flottants durant l'exposition. Le choix de maintenir les contacts flottants a été dicté par une contrainte expérimentale (porte-échantillon) et correspond à une condition de mesure parmi d'autres couramment rencontrée dans la littérature $[2,16]$. Les doses ont été calculées pour l'oxyde de grille à $10 \%$ près, compte tenu de l'éventuelle atténuation du faisceau par les différentes couches (verre protecteur au bore, couche de $\mathrm{Si}_{3} \mathrm{~N}_{4}$, plot d'aluminium et grille en polysilicium) déposées sur le film d'oxyde. Après exposition aux rayonnements ionisants. les structures de test ont été stockées $50 \mathrm{~h}$ à température ambiante afin d'éviter que les phénomènes rapides de relaxation électrique post-contrainte ne viennent perturber nos mesures. Durant ce temps d'attente, les contacts électriques ont été maintenus flottants. Des mesures successives effectuées à l'issue de ce délai nous ont permis de constater que la réponse électrique du dispositif sous test $n$ évoluait plus durant plusieurs heures.

L'exploitation des caractéristiques $3 \mathrm{CP}$ nécessite la connaissance de la relation entre la tension de grille $V_{\mathrm{G}}$ et le potentiel de surface $\Phi_{\mathrm{S}}[6,7]$. Cette courbe peut être délicate à obtenir dans le cas de structures à dopage non uniforme et/ou présentant une densité d'états d'interface élevée. Une discussion récente de ce problème a été proposée par Nicollian [1]. A noter que la présence supplémentaire d'états d'interface (ou proches de l'interface) ayant des constantes d'émission ou de capture relativement longues peut être à l'origine de phénomènes d'hystérésis. Dans le cadre ce ce travail, nous avons déterminé la relation $\Phi_{S}\left(V_{G}\right)$ à partir de mesures charge-tension statiques $\left(Q-V^{\prime}\right)$ réalisées directement sur la capacité grille-substrat du transistor en utilisant une variante de la méthode de Ziegler et Klausmann [8] décrite en détail dans la référence [4]. La source, le drain et le substrat d'une part, la grille d'autre part, forment 
les deux électrodes de la capacité (voir l'encart de la Fig. 1). La courbe $\Phi_{\mathrm{S}}\left(V_{\mathrm{G}}\right)$ est calculée numériquement par la méthode de Pierret et Panigraphi [9, 10] à partir d'une résolution itérative de l'équation de Poisson à une dimension qui tient compte du profil de dopage du semiconducteur [4]. Bien que relativement ancienne, cette méthode numérique présente l'avantage d'être facilement programmable et de converger rapidement dans le cas de dopages strictement croissants ou décroissants en fonction de la distance à l'interface. Nous avons montré, dans une précédente étude [4], que la prise en compte du profil de dopage est indispensable si l'on veut extraire avec une bonne précision, pour ce type de structures à dopage implanté, le potentiel de surface en fonction de la tension de grille. Un bon accord est généralement constaté entre les courbes $C-V$ expérimentale et théorique de structures vierges ou dégradées, comme le montre la figure 1 dans le cas d'une structure ayant subi une dose d'irradiation de $1 \mathrm{Mrad}$ au niveau de la couche d'oxyde. Une légère augmentation de la capacité réelle par rapport à la capacité théorique est toutefois observée vers le minimum des courbes : elle correspond à la variation avec $\Phi_{S}$ de la charge piégée dans les états d'interface et vraisemblablement à un effet dû aux capacités parasites latérales (source/grılle et drain/grille). Mais la bonne correspondance des flancs des courbes $C$ - $V$ mesurée et calculée témoigne de la validité de la relation $\Phi_{\mathrm{S}}\left(V_{\mathrm{G}}\right)$ ainsi déterminée [4].

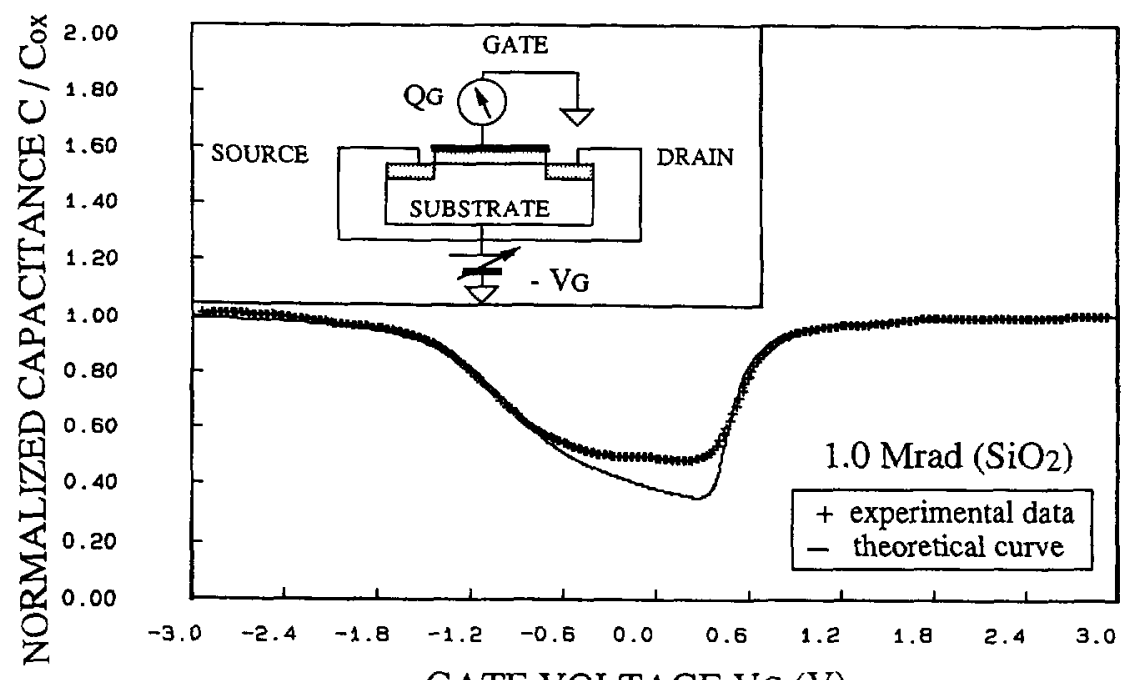

GATE VOLTAGE VG (V)

Fig. 1. - Courbe $C-V$ expérimentale (croix) obtenue par la méthode $Q-V$ statique sur un transistor MOS à canal $\mathrm{N}$ irradié. La courbe théorique (ligne continue) est calculée à partir de la résolution de l'équation de Poisson à une dimension pour un profıl de dopage non uniforme. Encart: Montage de base pour les mesures $Q-V$ statıques. La polarisation est appliquée au substrat, à la source et au drain ; la charge est mesurée sur la grille à l'aide d'un électromètre.

[Experimental $C \cdot V$ data (cross) obtained by static $Q-V$ method on an irradiated $\mathrm{N}$-channel MOS transistor. Theoretical curve (full line) is calculated from the resolution of Poisson's equation for the accurate nonconstant doping profile. Inset: Basic experimental set-up for $Q-V$ measurements. Bias is applied to the substrate, the source and the drain; the charge is measured on the gate with an electrometer.] 


\section{Résultats.}

3. 1 Pompage De Charge ClassiQue. - Après chaque irradiation, une étude préliminaire en pompage de charge classique est effectuée sur les dispositifs, afin d'évaluer l'incidence de la dégradation sur la réponse en pompage de charge. Nous présentons très brièvement, dans ce paragraphe, quelques résultats obtenus avec cette technique bien connue [11], dans le but de les comparer aux résultats déduits des mesures $3 \mathrm{CP}$. La figure 2 représente les caractéristiques $I_{\mathrm{cp}}\left(V_{\mathrm{gl}}\right)$ obtenues pour différentes doses d'irradiation. Après dégradation, la quasi-translation de la caractéristique initiale vers de plus fortes valeurs du courant pompé met en évidence la création uniforme de défauts électriquement actifs à la surface du canal : cette uniformité est sans doute due au fait que tous les contacts du dispositif sont maintenus à un potentiel flottant durant l'exposition aux rayonnements. Un faible élargissement des caractéristiques $I_{\mathrm{cp}}\left(V_{\mathrm{gl}}\right)$ vers les tensions négatives (queues de courant pour $V_{\mathrm{gl}}<-2,5 \mathrm{~V}$ ) est toutefois constaté (Fig. 2). Comme l'ont démontré Heremans et al. [12, 13], ces queues de courant sont dues aux fluctuations locales des tensions de seuil et de bandes plates aux extrémités du canal (les structures utilisées ici sont symétriques). Dans le cas des structures non dégradées, un profil de dopage non uniforme près de la source et du drain est très certainement à l'origine de la queue de courant. Dans le cas de structures irradiées, ces fluctuations semblent croître avec la dose d'exposition, ce qui signifie que les propriétés électriques du semiconducteur (dopage) et/ou de la couche d'oxyde (charges piégées) évoluent légèrement au cours de la dégradation. Ces résultats sont à rapprocher avec ceux très similaires obtenus dans le cas de dégradations électriques, par injection uniforme Fowler-Nordheim [14] ou par porteurs chauds [15].

L'étude en pompage de charge classique montre enfin que la moyenne géométrique $\langle\sigma\rangle$ des sections efficaces de capture diminue très nettement après irradiation des composants.

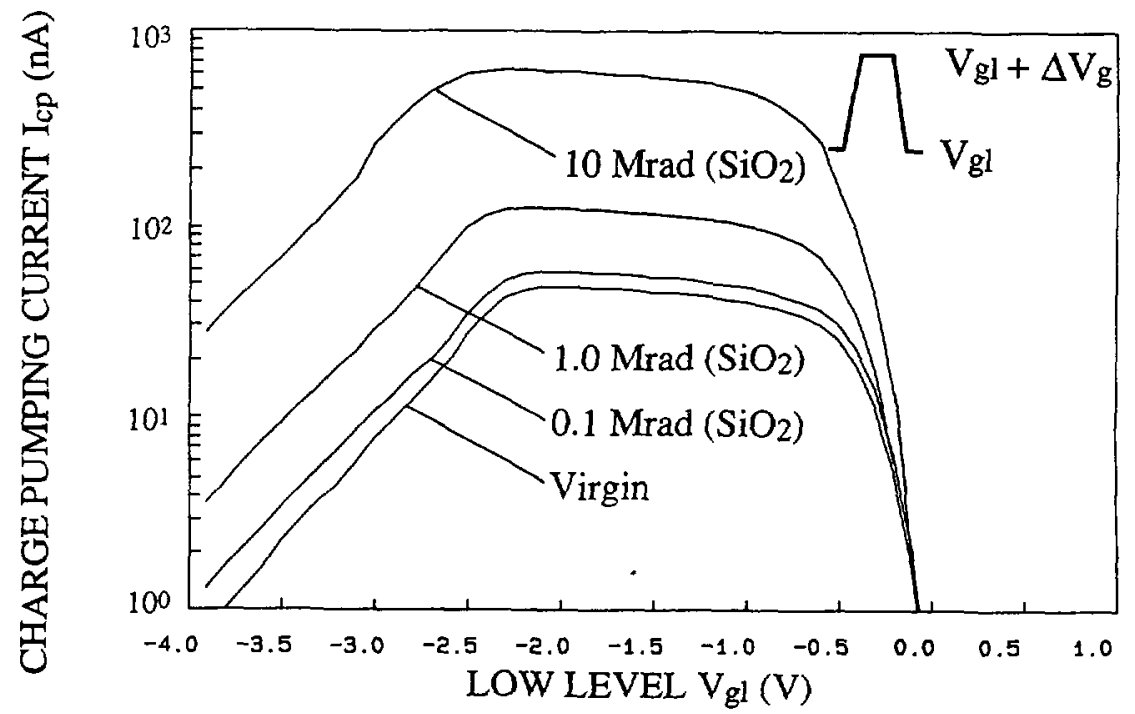

Fig. 2. - Caractéristiques $l_{\mathrm{cp}}\left(V_{\mathrm{gl}}\right)$ de pompage de charge pour différentes doses d'irradiation. Conditions de mesures : signal de grille trapézoïdal, $\Delta V_{\mathrm{g}}=3 \mathrm{~V}$, temps de descente et de montée $800 \mathrm{~ns}$. tension inverse de polarisation des jonctions source/substrat et drain/substrat $V_{\mathrm{R}}=0,2 \mathrm{~V}$.

$\left[I_{\mathrm{cp}}\left(V_{\mathrm{gl}}\right)\right.$ charge pumping characteristics for different doses of irradiation. Experimental conditions : trapezoidal pulses, $\Delta V_{\mathrm{g}}=3 \mathrm{~V}$, fall and rise time $800 \mathrm{~ns}$, reverse voltage at source and drain $\left.V_{\mathrm{R}}=0.2 \mathrm{~V}.\right]$ 
Constaté dans de nombreuses études antérieures [2, 16, 17], cette évolution dénote un changement global des propriétés électroniques «apparentes» de l'interface, en terme d'interaction avec les porteurs libres du semiconducteur. Dans le cas de nos échantillons par exemple, la valeur de $\langle\sigma\rangle$ passe de $9 \times 10^{-16} \mathrm{~cm}^{-2}$ avant dégradation à $2 \times 10^{-16} \mathrm{~cm}^{-2}$ pour une dose d'exposition de 1 Mrad et à $4 \times 10^{-17} \mathrm{~cm}^{-2}$ pour une dose de $10 \mathrm{Mrad}$. Néanmoins, $\langle\sigma\rangle$ est une valeur moyennée qui concerne uniquement une fraction des états de la bande interdite du semiconducteur: toute tentative d'interprétation physique de cette valeur peut donc s'avérer délicate et montre la nécessité de pouvoir connaître la répartition énergétique des sections efficaces.

3.2 Pompage de ChARge BASSE FRÉquence. - Des mesures en pompage de charge à 2 niveaux (signal de grille trapézoïdal) et à faibles fréquences (inférieures à $1 \mathrm{kHz}$ ) ont été proposées très récemment par Paulsen et al. [18] comme une technique possible de caractérisation des pièges d'oxyde proches de l'interface (quelqués nanomètres). Il est généralement admis que ces sites peuvent interagir avec le semiconducteur par un mécanisme tunnel, soit directement avec les bandes de valence ou de conduction du semiconducteur, soit indirectement via les états d'interface [18-21]. Nous avons mesuré la charge pompée par période du signal de grille $Q_{\mathrm{n}}=I_{\mathrm{cp}} / f$ en fonction de la fréquence $f$ des pulses, tous les autres paramètres du signal de grille étant fixés. En présence d'états d'interface rapides (ayant typiquement un temps de réponse inférieur à la microseconde), cette charge est indépendante de la fréquence de mesure : tous les états d'interface énergétiquement situés à l'intérieur de " la fenêtre de pompage » dans la bande interdite du semiconducteur, peuvent émettre ou capturer des porteurs et donc participer au cycle de pompage de charge. En revanche, la présence simultanée des deux types d'états se traduit par une charge pompée non constante dans le domaine des basses fréquences car les états lents peuvent participer au phénomène dès lors que la fréquence des pulses est compatible avec leur constante de temps. Autrement dit, la contribution des états lents se caractérise par l'apparition d'un courant pompé supplémentaire aux basses fréquences, ce qui implique donc une augmentation de la charge recombinée par cycle. La figure 3 représente la réponse d'un composant avant et après irradiation. On constate qu'après contrainte (dose de $10 \mathrm{Mrad}$ ). il y a une augmentation significative de $Q_{\mathrm{It}}$ à partir de $600 \mathrm{~Hz}$ environ. Cette fréquence de coupure peut être interprétée comme étant égale à l'inverse de la constante de temps du mécanisme régissant l'échange de porteurs entre le semiconducteur et les pièges d'oxyde les plus proches de l'interface. Une fréquence identique de $600 \mathrm{~Hz}$ est mesurée pour des échantillons irradiés avec une dose de $1 \mathrm{Mrad}$. Ces résultats sont en bon accord avec ceux obtenus par Paulsen et al. [18] concernant des transistors irradiés sous polarisation.

\subsection{POMPage DE ChaRge À trois niveAuX.}

3.3.1 Temps d'émission et sections efficaces. - Les figures $4 \mathrm{a}$ et $4 \mathrm{~b}$ représentent les réseaux de courbes $Q_{\mathrm{t}}\left(V_{3}, t_{3}\right)$ obtenus en pompage de charge à trois niveaux (régime d'émission d'électrons), respectivement pour un transistor non dégradé et pour un transistor irradié à $10 \mathrm{Mrad}$. Pour une tension donnée $V_{3}$ du niveau intermédiaire correspondant à une certaine position du niveau de Fermi dans la bande interdite du semiconducteur, on détermine la constante de temps de saturation de la charge recombinée par cycle. Ce temps $t_{\text {५at }}$ correspond au temps d'émission des états dont la position énergétique correspond à celle du niveau de Fermi (Fig. 4a) [7]. Connaissant la relation $\Phi_{\mathrm{S}}\left(V_{\mathrm{G}}\right)$, on peut donc déterminer la distribution énergétique des temps d'émission (Fig. 5) et calculer celle des sections efficaces de capture (Fig. 6) des pièges électroniques, dans le cadre du formalisme Schokley-Read-Hall (SRH) [22]. La figure 5 montre que pour une énergie donnée dans la bande interdite, 


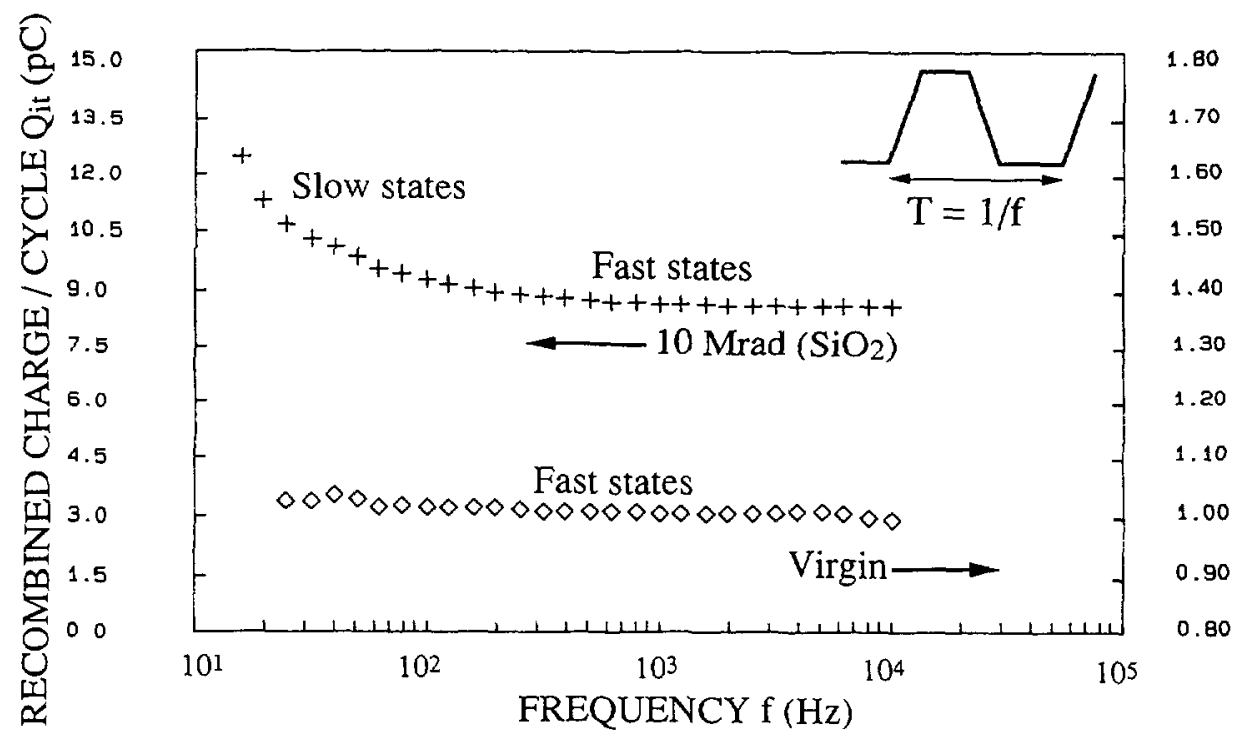

Fig. 3. - Charge recombınée par cycle $Q_{11}$ en fonction de la fréquence $f$ des pulses pour un dıspositif non irradıé et un dispositıf irradié à $10 \mathrm{Mrad}, V_{\mathrm{R}}=0,2 \mathrm{~V}$.

[Recombined charge per cycle $Q_{11}$ 'ersus the frequency $f$ of the pulses for a virgin device and for a $10 \mathrm{Mrad}$ irradıated device. $V_{\mathrm{R}}=0,2 \mathrm{~V}$.]

l'irradiation du composant entraîne une augmentation du temps d'émission des pièges mais que cette augmentation n est pas uniforme dans la partie inférieure de la bande interdite. En ce qui concerne les pièges à électrons, dans la partie supérieure de la bande interdite. une pente d'environ $q / k T$ est mesurée avant et après dégradation. Les temps d'émission après irradiation sont supérieurs d'environ une décade à ceux avant irradiation, ce qui se traduit par une diminution d'un facteur dix des sections efficaces (de $10^{-16}$ à $10^{-17} \mathrm{~cm}^{2}$ ). Il n'en est pas de même pour les pièges à trous dont les sections efficaces dépendent visiblement de la position énergétique des pièges avant et après irradiation. L'augmentation des temps d'émission après irradiation est quasi nulle pour les pièges proches de la bande de valence mais croît progressivement pour atteindre un facteur quatre, un peu en dessous du milieu de la bande interdite. Cette légère augmentation des temps d'émission entraîne donc une légère diminution des sections efficaces de capture. Cette différence de comportement des pièges à électrons et à trous n'est actuellement pas expliquée. D'après la figure 6 , le fait que les sections efficaces des pièges à électrons ne soient pas constantes avant irradiation, demeure inchangé après irradiation, ce qui pourrait laisser supposer que cette propriété est surtout intrinsèquement dépendante de la qualité initiale de l'interface, c'est-à-dire des conditions d'élaboration des structures de test. Dans de récents travaux concernant des transistors technologiquement semblables à ceux de cette étude mais ne provenant pas du même lot de fabrication, un résultat inverse a été observé, à savoir des sections efficaces constantes pour les pièges à trous et légèrement dépendantes de l'énergie pour les pièges à électrons [3]. Par ailleurs, comme l'ont montré Siergiej et al [23], des transistors ayant un canal fortement dopé en surface $\left(10^{17} \mathrm{~cm}^{-3}\right.$ ou plus) peuvent être le siège deffets quantiques influant sur les propriétés électroniques des pièges d'interface. En particulier, ces auteurs ont démontré qu'en utilisant une statistique SRH modifiée, tenant compte d'une quantification du bas de la bande de conduction en niveaux discrets, les sections efficaces de capture calculées pour les pièges à 

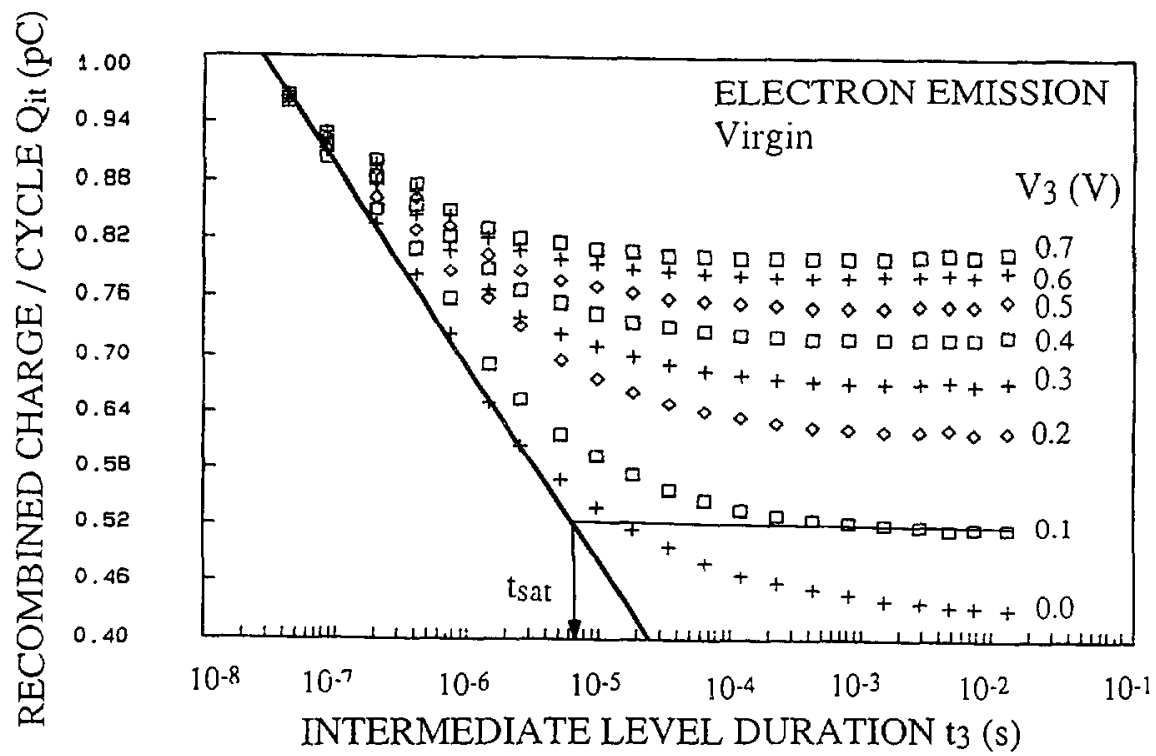

a)
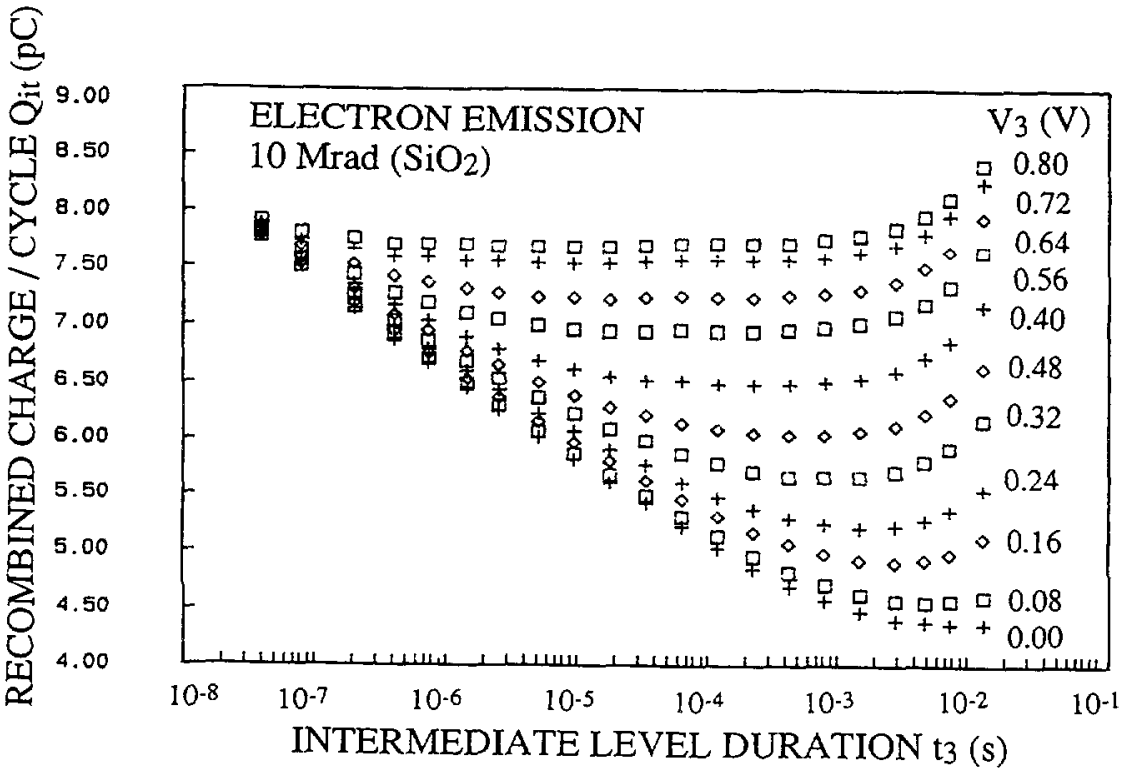

b)

Fig. 4. - Charge recombinée par cycle $Q_{11}$ en fonctıon de la durée $t_{3}$ et de la tensıon $V_{3}$ du niveau intermédiaire en régime d'émission d'électrons pour un transistor non irradié (a) et pour la même structure irradiée à $10 \mathrm{Mrad}$ (b). Le temps de saturation $t_{\mathrm{s}}$ est détermıné par construction graphıque pour chaque valeur de $V_{\mathrm{g}} . V_{\mathrm{gh}}=+3 \mathrm{~V}, V_{\mathrm{gl}}=-3 \mathrm{~V}$, temps de montée et de descente $20 \mathrm{~ns}, V_{\mathrm{R}}=0 \mathrm{~V}$.

[Recombined charge per cycle $Q_{11}$ as a function of third level parameters $t_{3}$ and $V_{3}$ in electron emission for a transistor before (a) and after (b) $10 \mathrm{Mrad}$ irradiation. Saturation time $t_{\mathrm{rat}}$ is graphically determined for each value of $V_{3} . V_{\mathrm{gh}}=+3 \mathrm{~V}, V_{\mathrm{gl}}=-3 \mathrm{~V}$, fall and rise times $20 \mathrm{~ns}, V_{\mathrm{R}}=0 \mathrm{~V}$.] 


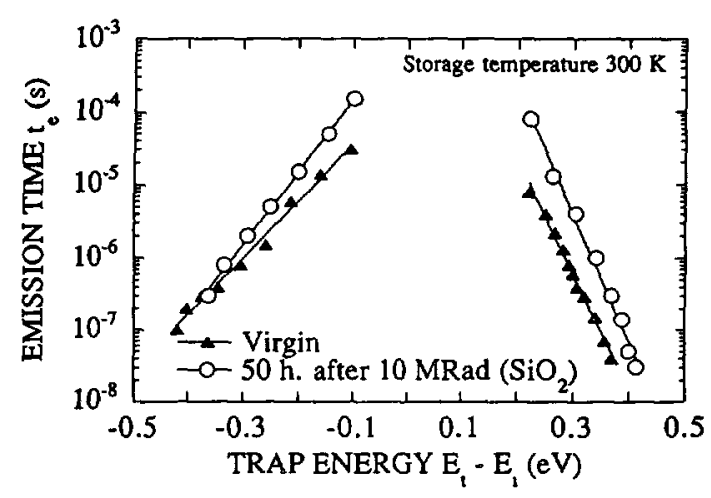

Fig.5.

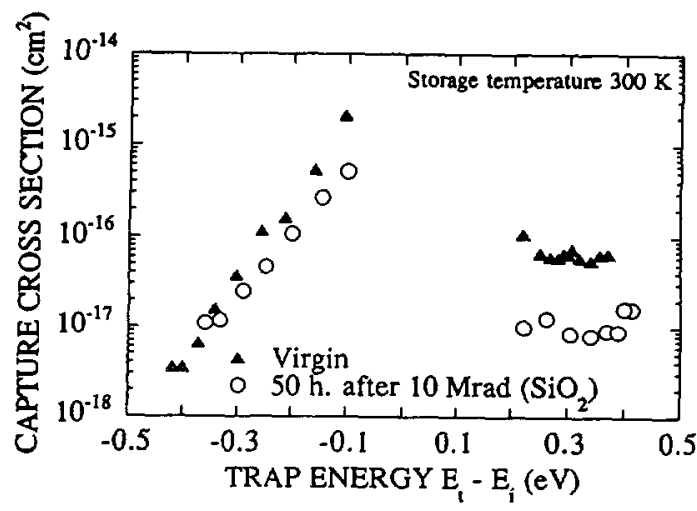

Fig. 6.

Fig. 5. - Distribution énergétique des temps d'émission dans la bande interdite du silıcium pour un dispositif non irradié (triangles) et pour un dispositif ayant subi une dose d'irradiation de 10 Mrad et un stockage de $50 \mathrm{~h}$ à $300 \mathrm{~K}$ (cercles).

[Energy distribution of electron and hole emıssion times in the silıcon bandgap for a nonirradiated device (triangles) and for a $10 \mathrm{Mrad}$ irradiated device after $50 \mathrm{~h}$ at $300 \mathrm{~K}$ (open circles).]

Fig. 6. - Sections efficaces de capture pour les pièges à électrons (respectivement à trous) obtenues en régime d'émission dans la partie supérieure (respectıvement inférieure) de la bande interdite du silicium pour un dispositif non irradié (triangles) et pour un dispositif ırradié à $10 \mathrm{Mrad}$ et stocké $50 \mathrm{~h}$ à $300 \mathrm{~K}$ avant la mesure (cercles).

[Electron (above midgap) and hole (blow midgap) capture cross sections obtained in emission regime as a function of the energy-trap position in silicon bandgap for a nonirradiated device (triangles) and for a $10 \mathrm{Mrad}$ irradiated device after $50 \mathrm{~h}$ at $300 \mathrm{~K}$ (open circles).]

électrons étaient supérieures d’une décade à celles extraites par la théorie classique. Dans le même article, une fluctuation similaire des sections efficaces est mentionnée pour des transistors relatifs à deux études différentes, ce qui tend à confirmer finalement que les propriétés électroniques des pièges sont fortement dépendantes des dispositifs, c'est-à-dire de la technologie de fabrication. La conclusion est donc qu il faut être extrêmement prudent sur l'évolution des paramètres d'émission des états d'interface, après irradiation, même si l'ensemble des travaux, y compris cette étude par pompage de charge à trois niveaux, tend à démontrer une diminution non uniforme des sections efficaces de capture dans la bande interdite du silicium.

3.3.2 Densité d'états d'interface. - La distribution énergétique $D_{\mathrm{It}}(E)$ des états d'interface est déterminée à partir des courbes $Q_{\text {It }}\left(V_{3}\right)$ en régime d'émission de porteurs (i.e. à l'équilibre) et pour une durée donnée du temps intermédiaire $t_{3}$ qui fixe la fenêtre des temps d'émission de la technique [24]. La figure 7 représente ces courbes dans le cas d'une première structure non dégradée et d'une deuxième exposée à une dose de $1 \mathrm{Mrad}$. La déformation et le décalage vers le haut du graphique des courbes obtenues après irradiation résultent d'une augmentation sensible du nombre d'états d'interface, plus importante néanmoins pour les pièges à électrons (courbe $1, V_{3}>0$ ) que pour les pièges à trous (courbe $2, V_{3}<0$ ). Sur la figure 8 sont représentés les spectres $D_{\mathrm{tt}}(E)$ obtenus pour différentes doses d'exposition, toujours après un temps d'attente de $50 \mathrm{~h}$ à température ambiante. Le spectre de référence (structure vierge) met 


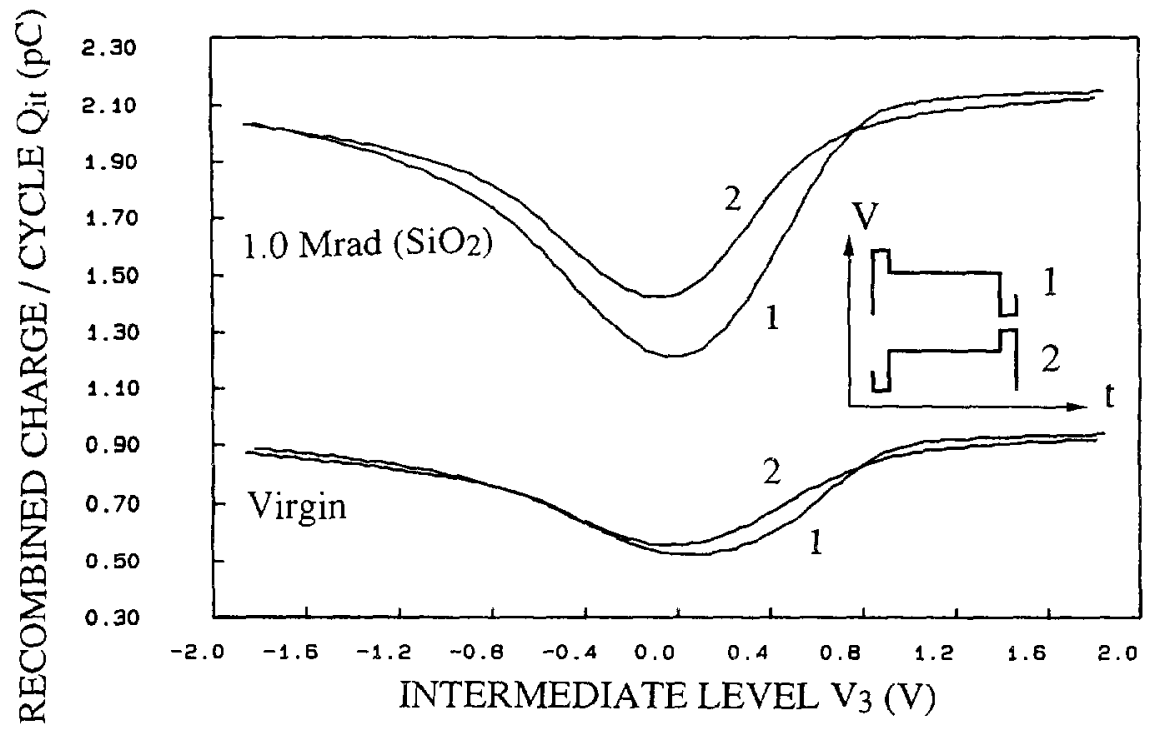

Fig. 7. - Charge recombinée par cycle $Q_{11}$ en fonction de la tension $V_{3}$ du nıveau intermédiaire pour un transistor non irradié et pour un transistor irradié à $1 \mathrm{Mrad}$. Les courbes numérotées 1 (respectivement 2) se rapportent à l'émission d'électron et à la capture de trous (respectivement émission de trous et capture d'électrons). $V_{\mathrm{gh}}=+3 \mathrm{~V}, V_{\mathrm{gl}}=-3 \mathrm{~V}$, temps de montée et de descente $20 \mathrm{~ns}, t_{\mathrm{1}}=6 \mathrm{~ms}, V_{\mathrm{R}}=0 \mathrm{~V}$.

[Recombined charge per cycle as a function of $V_{3}$ for a virgin device and for a 1 Mrad irradiated device. Curves labelled 1 : electron emission and hole capture regimes; curves labelled $2:$ hole emission and electron capture regimes. $V_{\mathrm{gh}}=+3 \mathrm{~V}, V_{\mathrm{gl}}=-3 \mathrm{~V}$, transition times $=20 \mathrm{~ns}, t_{3}=6 \mathrm{~ms}, V_{\mathrm{R}}=0 \mathrm{~V}$.]

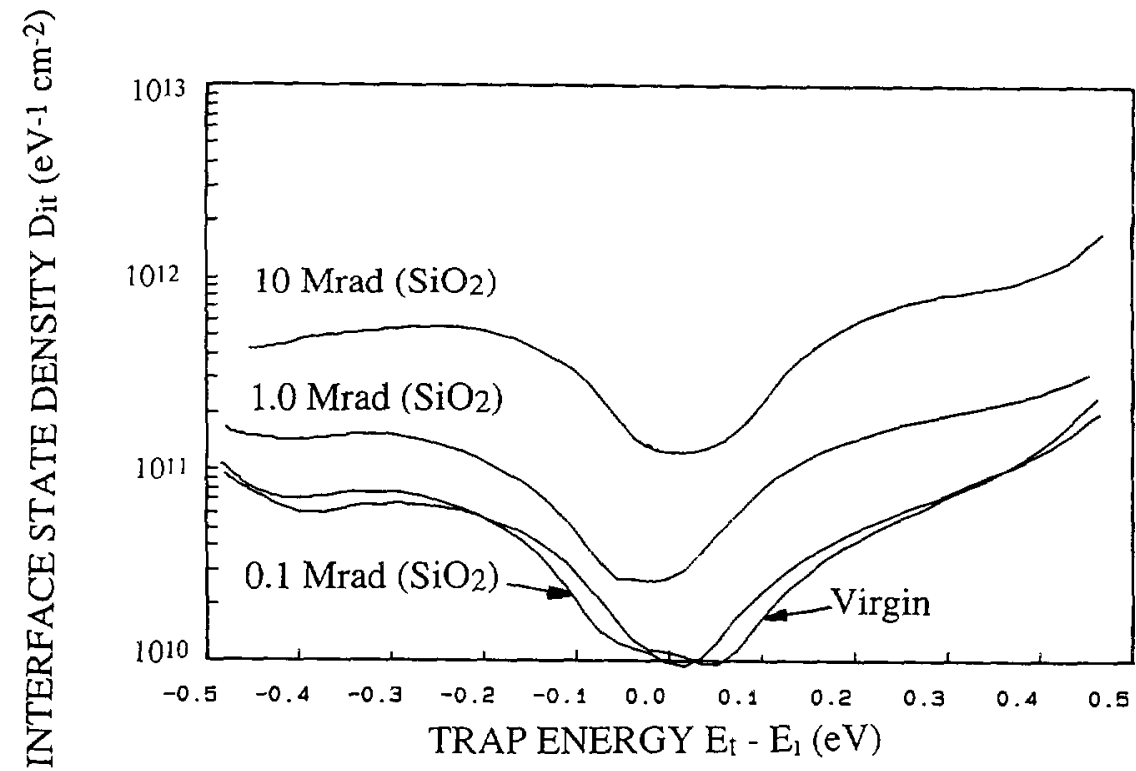

Fig. 8. - Spectres de la densité d'états d'ınterface dans la bande interdıte du sılicium obtenues pour différentes doses d'irradıatıon à partir des courbes $Q_{11}\left(V_{3}\right)$, la valeur de $t_{3}$ étant fixée à $6 \mathrm{~ms}$.

[Energy distributions of interface state density in silicon bandgap obtained for different irradiated devices from $Q_{11}\left(V_{3}\right)$ curves, the value of $t_{3}$ being fixed to $6 \mathrm{~ms}$.] 
en évidence deux bosses centrées approximativement autour de $E_{\mathrm{v}}+0,35 \mathrm{eV}$ et de $E_{\mathrm{v}}+0,75 \mathrm{eV}$ qui sont probablement dues à la présence initiale de défauts d'interface inhérents au procédé de fabrication. Au fur et à mesure que la dose d'exposition croît, l'allure générale des spectres se modifie notablement. On assiste à une nette augmentation de la densité d'états d'interface moyenne et surtout à un renforcement des deux bosses précédentes, ce qui indique une importante création de défauts d'interface dans la bande interdite du silicium et particulièrement près de $E_{\mathrm{v}}+0,35 \mathrm{eV}$ et de $E_{\mathrm{v}}+0,75 \mathrm{eV}$. Cependant, le pic à $E_{\mathrm{v}}+0,75 \mathrm{eV}$ semble être moins large que le pic à $E_{\mathrm{v}}+0,35 \mathrm{eV}$. Nous avons cherché à confronter nos résultats avec ceux de la littérature récente qui sont, à ce sujet, relativement nombreux et variés [25-33]. Si la plupart des auteurs aboutissent aux mêmes conclusions en ce qui concerne le nombre et la position énergétique $\left(E-E_{\mathrm{v}}=(0,35 \pm 0,1) \mathrm{eV}\right.$ et $E-E_{\mathrm{v}}=$ $(0,70 \pm 0,05) \mathrm{eV})$ des zones de la bande interdite du silicium (orienté $\langle 100\rangle)$ où se créent majoritairement le plus de défauts, l'évolution de ces deux pics après contrainte est visiblement un problème plus complexe. Les différents comportements de l'interface après dégradation résultent probablement de l'influence. d'une part, des paramètres technologiques de fabrication sur les défauts électriquement actifs et, d'autre part, des conditions de dégradation et de mesure.

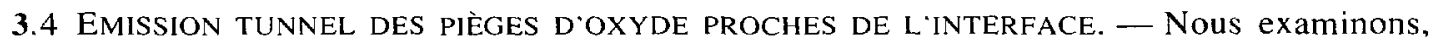
dans ce paragraphe, la contribution des pièges d'oxyde proches de l'interface à la réponse en pompage de charge à trois niveaux d'un échantillon irradié. Contrairement au cas des structures non dégradées, les courbes $Q_{1 \mathrm{t}}\left(t_{3}\right)$ de la figure $4 \mathrm{~b}$ présentent une augmentation inhabituelle de la charge recombinée par cycle pour des durées du niveau intermédiaire supérieures à une milliseconde. Un tel comportement en basse fréquence est à rapprocher avec celui observé par pompage de charge classique (Sect. 3.2). Toutefois, les mesures 3CP donnent des informations plus précises que celles obtenues en pompage $2 \mathrm{CP}$ basse fréquence car elles permettent d'établir que la réponse des états lents est contrôlée par un mécanisme d'émission. En effet seule la durée du régime d'émission varie lors de l'acquisition des courbes $Q_{1 \mathrm{t}}\left(t_{3}\right)$ (Fig. 4b). Grâce à la procédure d'acquisition à trois niveaux que nous avons proposée [3|, il est possible d'observer directement le début du régime d'émission des états lents via les états d'interface rapides, et donc de mesurer les constantes de temps du mécanisme régissant l'échange des porteurs entre les états lents, situés à proximité de l'interface, et les états rapides, localisés à l'interface même. D'après la figure $4 \mathrm{~b}$, ces constantes de temps sont comprises entre 1 et $10 \mathrm{~ms}$ pour $0,80 \mathrm{~V}<V_{3}<0,24 \mathrm{~V}$ et supérieures à $10 \mathrm{~ms}$ pour $V_{3}<0,16 \mathrm{~V}$. Cette évolution des constantes de temps pour les faibles valeurs de $V_{3}$ n'est cependant pas évidente. En effet, le régime de saturation des états rapides proches du milieu de la bande interdite du semiconducteur $n$ est pas encore atteint : la contribution des états lents peut être alors contrebalancée par la diminution de la charge pompée dans les états rapides. Nous retiendrons donc une valeur moyenne de $10 \mathrm{~ms}$ pour la constante de temps du processus tunnel piège à piège régissant l'échange de porteurs entre les pièges d'oxyde et le semiconducteur via les états d'interface.

Les différents modèles de mécanisme tunnel disponibles dans la littérature établissent une correspondance directe entre la distance des pièges par rapport à l'interface et la constante de temps du processus tunnel [34-37]. D'après les travaux de plusieurs auteurs [37, 38], on peut raisonnablement considérer que ces défauts d'oxyde sont localisés énergétiquement dans une zone d'environ $100 \mathrm{meV}$ au-dessous de la bande de conduction du semiconducteur. Connaissant la densité moyenne d'états d'interface rapides $\left(D_{1 t} \approx 7 \times 10^{11} \mathrm{eV}^{-1} \mathrm{~cm}^{-2}\right)$, le modèle de Roy [37] donne une distance moyenne des pièges par rapport à l'interface comprise entre 15 et $20 \AA$, ce qui est en bon accord avec les valeurs obtenues par d'autres techniques de mesure sur des composants irradiés ou dégradés électriquement $[18,37,38]$. 
3.5 DisTRIBUtion ÉNERGÉTIQUE DES ÉTATS LENTS. - Nous proposons, dans ce paragraphe, une nouvelle manière d'accéder à la répartition énergétique des états proches de l'interface, que nous avons qualifié d'états lents en pompage de charge [39], dans la bande interdite du semiconducteur. Cette distribution de pièges est directement déduite de la différence entre deux mesures $3 \mathrm{CP}$, pour deux durées distinctes $t_{3}^{\prime}$ et $t_{3}^{\prime \prime} \mathrm{du}$ niveau intermédiaire. $t_{3}^{\prime}$ et $t_{3}^{\prime \prime}$ définissent une fenêtre de temps d'émission pour un niveau $V_{3}$ fixé : la première valeur $t_{3}^{\prime}$ (borne inférieure de la fenêtre d'émission) est choisie de manière à ne prendre en compte que la contribution des états rapides; la seconde. $t_{3}^{\prime \prime}$ (borne supérieure). permet aux états lents de participer au phénomène de pompage de charge. La différence des deux signaux de pompage de charge (charge ou courant), mesurés respectivement pour $t_{3}=t_{3}^{\prime}$ et pour $t_{3}=t_{3}^{\prime \prime}$ correspond à la réponse des états situés dans cette fenêtre de temps d'émission. Le balayage de la fenêtre d'émission dans la bande interdite du semiconducteur s'effectue tout simplement en faisant varier la tension $V_{3}$ du niveau intermédiaire. Le calcul de la densité d'états lents à partir de la différence 'des deux charges $Q_{1 \mathrm{t}}$ mesurées pour $t_{3}=t_{3}^{\prime}$ et pour $t_{3}=t_{3}^{\prime \prime}$ est alors immédiat [40]. Sur la figure 9 est représentée la distribution de ces états proches de l'interface obtenue pour un composant irradié avec une fenêtre d'émission s'étendant de $1,2 \mathrm{~ms}$ à $12 \mathrm{~ms}$. Deux pics de densité d'états lents apparaissent nettement aux environs de $E_{\mathrm{y}}+0,33 \mathrm{eV}$ et de $E_{\mathrm{v}}+0,70 \mathrm{eV}$. Ces résultats sont à comparer aux récents travaux de Druijf et al. [32] qui mettent en évidence deux pics d'états lents à $E_{\mathrm{v}}+0,25 \mathrm{eV}$ et à $E_{\mathrm{v}}+0,65 \mathrm{eV}$ bien que la gamme des constantes de temps de ces pièges soit, dans ce cas, beaucoup plus étendue que celle explorée par pompage de charge, compte-tenu du caractère statique des mesures charge-tension. Notons enfin que la courbe de la figure 9 permet d'apprécier la sensibilité de notre méthode aux états lents. Nous évaluons celle-ci à environ $10^{10} \mathrm{eV}^{-1} \mathrm{~cm}^{-2}$ pour des temps d'émission inférieurs ou égaux à la dizaine de millisecondes.

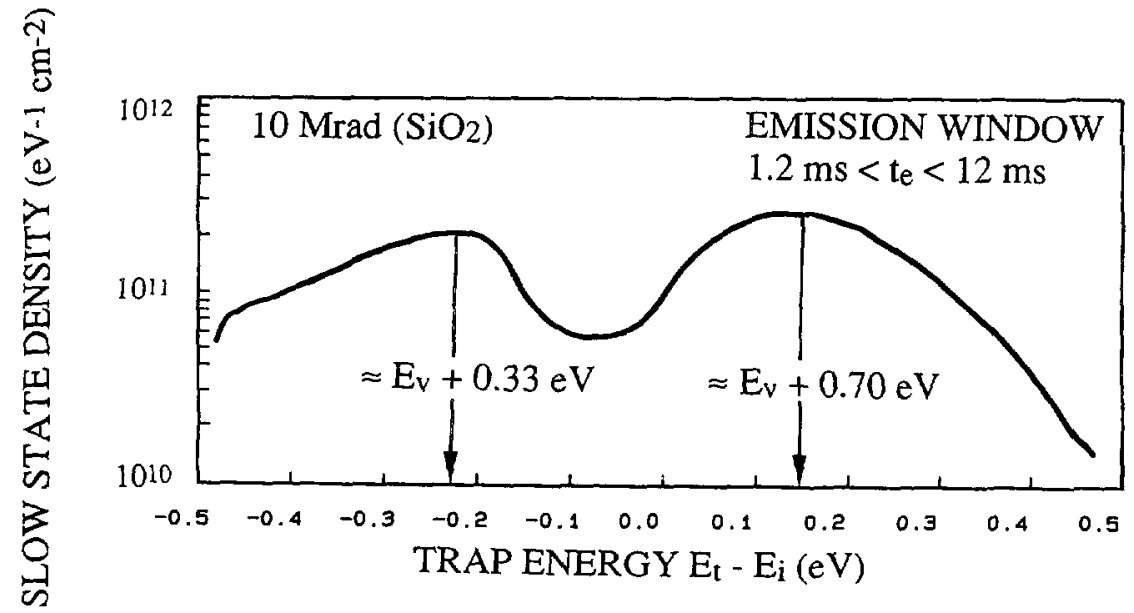

Fig. 9. - Distribution énergétique de la densité d’états lents dont les temps d’émission sont compris entre $t_{3}^{\prime}=1,2$ et $t_{3}^{\prime \prime}=12 \mathrm{~ms}$. Cette courbe est calculée à partir de mesures en pompage de charge à trois niveaux, pour deux valeurs distinctes $t_{3}^{\prime}$ et $t_{3}^{\prime \prime}$ de la durée du niveau intermédiaire en régime d'émission.

[Energy distribution of slow state density for emission times between $t_{3}^{\prime}=1.2$ et $t_{3}^{\prime \prime}=12 \mathrm{~ms}$. This curve is calculated from three-level charge pumping measurements, for two distinct values $t_{3}^{\prime}$ and $t_{3}^{\prime \prime}$ of the intermediate level in emission regime.] 


\section{Conclusion.}

La réponse électrique du système $\mathrm{Si}-\mathrm{SiO}_{2}$, après exposition à des rayonnements ionisants, a été étudiée par différentes approches de pompage de charge dans des transistors MOS submicroniques. Les principaux résultats de cette étude sont les suivants :

i) L'évolution des paramètres des états d'interface a été étudiée par la technique de pompage de charge à trois niveaux, simultanément dans les parties inférieures et supérieures de la bande interdite du semiconducteur. Nous avons montré que si leur évolution est conforme aux résultats antérieurs obtenus par d'autres techniques, en particulier pour la densité d'états d'interface après irradiation, la répartition énergétique des sections efficaces de capture après contrainte $\mathrm{n}$ est pas uniforme sur toute la bande interdite du silicium; elle apparaît comme extrêmement dépendante des paramètres technologiques des dispositifs ainsi que des conditions de dégradation et de mesures.

ii) Des mesures en pompage de charge classique nous ont permis de détecter la présence des défauts d'oxyde proches de l'interface en réalisant une étude en fréquence de la charge recombinée par période du signal de grille. Ces résultats ont été comparés à ceux obtenus par pompage de charge à trois niveaux.

iii) En définissant par différence, dans la bande interdite du semiconducteur, une fenêtre de pompage de charge à faible vitesse d'émission, nous avons démontré que cette technique offre la possibilité de déterminer une répartition énergétique des états proches de l'interface (qualifiés d'états lents en pompage de charge) dans la bande interdite du semiconducteur. Ces différentes approches à très basse fréquence des techniques de pompage de charge ouvrent de nouvelles perspectives pour l'étude des processus d'émission ou de capture à longues constantes de temps et nous semblent particulièrement prometteuses sur le plan de la caractérisation électrique des dispositifs fortement submicroniques.

\section{Remerciements.}

Cette étude a pu être menée à bien grâce à IBM Microelectronics, Corbeil-Essonnes. Nous tenons à remercier Louis-Marie Gaborieau (Failure Analysis, Advanced CMOS Line) pour son soutien et sa disponibilité tout au long de ce travail. Nous tenons également à remercier Carole Plossu, Frédéric Seigneur et Christophe Raynaud pour les nombreuses discussions que nous avons eues ensemble durant cette étude.

\section{Bibliographie}

[1] Nicollian E. H., Electrical Characterization of defects created in the $\mathrm{S}_{1}$-SiO, system by ionizing radiations, J. Electron. Mater. 21 (1992) 721-729.

[2] Ma T. P., Generation and transformation of interface traps in MOS structures. Microelectron. Eng. 22 (1993) 197-200.

[31 Autran J. L., Balland B., A new three-level charge pumpıng method for accurate determınation of interface-trap parameters in metal-oxide-semiconductor field-effect transistors, Rev $S c i$. Instrum. 65 (1994) 2141-2142.

[4] Autran J. L., Balland B., Gaborieau L. M., Development of an interface trap measurement system using 3-level charge pumpıng for submicrometer MOSFETs, IBM I Res. Development, à paraître (1994).

[5] Autran J. L., Djahli F., Balland B., Plossu C., Gaborieau L. M., Three-level charge pumping on submicronic MOS transistors, Solid State Commun 84 (1992) 607-611. 
[6] Tseng W. L., A new charge pumping method of measuring $\mathrm{Si}^{-\mathrm{SiO}_{2}}$ interface states. J. Appl Phys. 62 (1987) 591-599.

[7] Saks N. S., Ancona M. G., Determination of interface trap capture cross sections using three-level charge pumping. IEEE Trans. Electron Devices 37 (1990) 1057-1058.

[8] Ziegler K., Klausmann E., Static technique for precise measurements of surface potential and interface state density in MOS structures, Appl. Phys. Lett. 26 (1975) 400-402.

[9] Pierret R. F., Panigraphi G.. Solution of Poisson's equation appropriate for semiconductors with nonconstant impurity profiles, J. Appl. Phys. 41 (1970) 2260-2261.

[10] Panigraphı G., Numerical calculation of low-frequency capacitance/voltage curves of MOS capacitors with nonconstant doping profiles, Electron. Lett. 9 (1973) 43-44.

[11] Groeseneken G., Maes H. E., Beltran N., de Keersmaecker R. F., A reliable approach to chargepumping measurements in MOS transistors, IEEE Trans. Electron Devices 31 (1984) 42-53.

[12] Heremans P.. Bellens R., Groeseneken G., Maes H. E., Consistent model for the hot-carrier degradation in n-channel and p-channel MOSFET's, IEEE Trans. Electron Devices 35 (1988) 2194-2209.

[13] Heremans P., Witters J., Groeseneken G., Maes H. E., Analysis of the charge pumping technıque for the evaluation of MOSFET degradation. IEEE Trans. Electron Devices 36 (1989) 1318 1335.

[14] Nguyen-Duc C., Ghibaudo G., Balestra F., MOSFET degradation studied by low frequency noise, charge pumping and static $I(V)$ measurements, Physica Status Solidi A 126 (1991) 553-560.

[15] Vuillaume D., Caractérisation et propriétés physiques des défauts induits dans les transistors MOS submicroniques par injections de porteurs chauds, J. Phys. III France 2 (1992) 777-804.

[16] Chen W., Ma T. P., Time evolution of capture cross sections of radiation-induced $\mathrm{Si} / \mathrm{SiO}_{2}$ interface traps studied by single-frequency ac conductance technique, J. Appl. Phys. 70 (1991) 860-866.

[17] Chen W., Balasinski A., Ma T. P., Evolution of capture cross section of radiation induced interface traps in MOSFETs studied by a rapid charge pumping techmque, IEEE Trans. Nuclear Sc. 39 (1992) 2152-2157.

[18] Paulsen R. E., Siergiej R. R., French M. L.. White M. H., Observation of near-interface oxide traps with the charge pumping technique, IEEE Electron Device Lett. 13 (1992) 627-629.

[19] Kirton M. J., Uren M. J., Collins S., Schulz M., Karmann A., Scheffer K., Individual defects at the $\mathrm{Si}_{-} \mathrm{SiO}_{2}$ interface, Semiconductor Sct. Technol. 4 (1989) 1116-1126.

[20] Fleetwood D. M., Border traps in MOS devices, IEEE Trans. Nuclear Sci. 39 (1992) 269.

[21] Fleetwood D. M., Winokur P. S., Reber R. A., Meisenheimer T. L., Schwank J. R., Shaneyfelt M. R., Riewe L. C., Effects of oxide traps, interface traps and border traps on metal-oxidesemiconductor devices, J. Appl. Phys. 73 (1993) 5058-5074.

[22] Ancona M. G., Saks N. S., Numerical simulation of 3-level charge pumping, I. Appl. Phys. 71 (1992) 4415-4421.

[23] Siergiej R. R., White M. H. and Saks N., Theory and measurement of quantization effects on Si$\mathrm{SiO}_{2}$ interface trap modeling, Solid-State Electron. 35 (1992) 843-854.

[24] Autran J. L., Seigneur F., Plossu C., Balland B., Characterization of Si-SiO 2 interface states : comparison between different charge pumping and capacitance techniques, J. Appl. Phys. $\mathbf{7 4}$ (1993) 3932-3935.

[25] Stahlbush R. E., Lawrence R. K., Hughes H. L., Saks N. S., Annealing of total dose damage : redistribution of interface state density on $\langle 100\rangle,\langle 110\rangle$ and $\langle 111\rangle$ orientation silicon, IEEE Trans. Nuclcar Sci. 35 (1988) 1192-1196.

[26] Barnes C., Zietlow T., Nakamura K.. The application of deep level transient spectroscopy to the measurement of radiation-induced interface state spectra, IEEE Trans. Nuclear Sci. 35 (1988) 1197-1202.

[27] Da Silva E. F., Nishioka Y., Ma T.P., Radiation response of MOS capacitors containing fluorinated oxides, IEEE Trans. Nuclear Sci. 34 (1987) 1190-1195.

[28] Nishioka Y., Da Silva E. F.. Ma T. P.. Radiation-induced interface traps in $\mathrm{Mo} / \mathrm{SiO}_{2} / \mathrm{Si}$ capacitors, IEEE Trans. Nuclear Sci. 34 (1987) 1166-1171.

[29] Wang Y., Ma T. P., Barker R. C., Orientation dependence of interface-trap transformation, IEEE Trans. Nuclear Sci. 36 (1989) 1784-1791. 
[30] Wang X. W., Wang Y., Wang D., Ma T. P., Improvement of radiation hardness due to aging of fluorinated and chlorinated $\mathrm{SiO}_{2} / \mathrm{Si} \mathrm{MOS}$ capacitors, IEEE Trans. Nuclear Sci. 39 (1992) 2252-2256.

[31] Kelleher A., Heyns M., Wulf F., Lane W., Ionizing radiation effects on phosphorus implanted $\mathrm{N}^{+}$ poly gate MOS capacitors with thin gate oxides, IEEE Trans. Nuclear Sci. 39 (1992) 20042011 .

[32] Druijf K. G., De Nijs J. M. M., van der Drift E., Granneman E. H. A., Balk P., Charge exchange mechanisms of slow states in $\mathrm{Si} / \mathrm{SiO}_{2}$, Microelectron. Eng. 22 (1993) 231-234.

[33] McWhorter P. J., Fleetwood D. M., Pastorek R. A., Comparison of MOS capacitor and transistor postirradiation response, IEEE Trans. Nuclear Sci. 36 (1989) 1792-1799.

[34] Balland B., Pinard P., Interfacial tunnel interaction in MOS elements in depletion, Physica Status Solidi A 47 (1978) 251-256.

[35] Vuillaume D., Bourgoin J. C., Lannoo M., Oxide traps in Si-SiO $\mathrm{S}_{2}$ structures characterized by tunnel emission with deep level transient spectroscopy, Physical Rev, B 34 (1986) 1171-1183.

[36] Hwang N., Or B. S. S., Forbes L., Tunneling and thermal emission of electrons from a distribution of deep traps in $\mathrm{SiO}_{2}$, IEEE Trans. Electron Devices 40 (1993) 1100-1103.

[37] Roy A., Characterization and modelling of charge trapping and retention in novel multi-dielectric nonvolatile semiconductor memory devices, Ph. D. dissertation, Lehigh University, 1989 (unpublished).

[38] Lakhdari H., Vuillaume D., Bourgoin J. C., Spatial and energetic distribution of $\mathrm{Si}_{-} \mathrm{SiO}_{2}$ nearinterface states, Physical Rev. B 38 (1988) 13124-13132.

[39] Fletwood D. M., Shaneyfelt M. R., Schwank J. R., Estimating of oxide-trap, interface-trap and border-trap densities in metal-oxide-semiconductor transistors. Appl. Phys. Lett. 64 (1994) 1965-1967. La remarque de la référence [16] est intéressante car elle montre que les qualificatifs d'états rapides ou lents sont relatifs à la technique de mesure utilisée. Dans le cas des mesures en pompage de charge, les états qualifiés de «lents» ont des constantes de temps beaucoup plus faibles (de l'ordre de la dizaine, voire de la centaine de millisecondes) que celles des états lents mis en évidence, par exemple, lors du survi de l'hystérésis des courbes capacitétension (constantes de temps généralement supérieures à la seconde).

[40] Autran J. L., Balland B.. Plossu C.. Seigneur F., Gaborieau L. M., Application du pompage de charge à trois niveaux aux transistors MOS submicroniques, I Phys. III France 3 (1993) 3345 . 